\title{
On the transfer reducibility of certain Farrell-Hsiang groups
}

\author{
CHRISTOPH WINGES
}

\begin{abstract}
We show how the existing proof of the Farrell-Jones conjecture for virtually poly- $\mathbb{Z}-$ groups can be improved to rely only on the usual inheritance properties in combination with transfer reducibility as a sufficient criterion for the validity of the conjecture.
\end{abstract}

$18 \mathrm{~F} 25 ; 54 \mathrm{H} 25,55 \mathrm{U} 10$

\section{Introduction}

The Farrell-Jones conjecture predicts that a certain assembly map

$$
\alpha_{\mathcal{V C} y c}^{G}: H_{n}^{G}\left(E_{\mathcal{V C} y c} G ; \mathbb{K}_{\mathcal{A}}^{-\infty}\right) \rightarrow K_{n}\left(\mathcal{A} *_{G} G / G\right)
$$

is an isomorphism for all discrete groups $G$ and small additive $G$-categories $\mathcal{A}$; there is also an $L$-theoretic version of the conjecture which replaces the nonconnective $K$-theory spectrum of Pedersen and Weibel [22] by Ranicki's ultimate lower $L$-groups (see eg Ranicki [24, Section 17] and Carlsson and Pedersen [9, Section 4]). These conjectures have received a lot of attention since their introduction by Farrell and Jones [14] due to their intimate relation with other prominent conjectures such as the Borel and Novikov conjectures. See Lück and Reich [16] for a survey.

While the conjectures are still wide open in general, substantial progress has been made on the question in which special cases the conjectures hold. Among the most notable classes of examples, one finds hyperbolic (see Bartels, Lück and Reich [7]) and CAT(0)-groups (see Bartels and Lück [4] and Wegner[28]), virtually poly- $\mathbb{Z}-$ groups [2], lattices in virtually connected Lie groups (see Bartels, Farrell and Lück [2] and Kammeyer, Lück and Rüping [15]), a large number of linear groups (see Bartels, Lück, Reich and Rüping [8]), and solvable groups (see Wegner [29]).

Normally, the proofs can be broken down into several steps. Starting from the most general case, one uses certain inheritance properties of the conjectures to reduce the proof to simpler instances. These are then dealt with by proving that the groups under consideration satisfy the assumptions of an abstract criterion which has been independently shown to imply the conjecture. 
These criteria include two prototypical examples. First, there is the notion of transfer reducibility which was used to prove the case of hyperbolic groups and then generalized to also cover CAT(0)-groups. Second, the property of being a Farrell-Hsiang group (an abstraction of the arguments employed by Farrell and Hsiang in $[11 ; 12 ; 13]$ and further exploited by Quinn [23]) was considered to obtain proofs for virtually poly- $\mathbb{Z}$-groups.

The goal of the present article is to show that the $K$ - and $L$-theoretic Farrell-Jones conjectures for virtually poly- $\mathbb{Z}$-groups [2, Theorem 1.1$]$ can be deduced relying entirely on transfer reducibility as a sufficient criterion, bypassing any use of the Farrell-Hsiang method (ie the results of Bartels and Lück in [5]). This illustrates that being transfer reducible is not a concept inherently concerned with nonpositive curvature conditions, as the original examples of transfer reducible groups, namely hyperbolic and CAT(0)-groups, might suggest.

As a consequence of our results, the proofs of the Farrell-Jones conjecture for lattices in virtually connected Lie groups by Bartels, Farrell and Lück [2, Theorem 1.2] and Kammeyer, Lück and Rüping [15] also become independent of the Farrell-Hsiang method (eg the proof of Theorem 1.2 in [2] only requires the validity of the conjecture for CAT(0)-groups as additional input).

It should be pointed out that the results of this article cannot be considered a simplification of the existing proofs, as the arguments that go into the verification of the Farrell-Hsiang condition still have to be employed. However, it does serve the purpose of unifying the existing proofs; the results of [5] are not needed anymore.

Virtually cyclic groups form a notable exception to the slogan that "Farrell-Hsiang groups are transfer reducible". The reduction to the family of (possibly infinite) hyperelementary groups presented by Quinn [23, Proposition 3.1.1] and Bartels, Farrell and Lück [2, Section 8] cannot be obtained with the methods of this article.

We proceed as follows: In Section 2 and Section 3, we formulate a strengthening of the Farrell-Hsiang condition and prove that all groups which satisfy this stronger condition are transfer reducible in a very strict sense. Once this has been done, we give a quick review of the structure of the proof of [2, Theorem 1.1] in Section 4. This serves the purpose of singling out all instances of the Farrell-Hsiang condition that appear in the proof. In the remaining sections Section 5 and Section 6, we will present proofs that the classes of groups isolated in Section 4 all satisfy our stronger version of the Farrell-Hsiang condition. The appendix reviews a theorem of Oliver [19] concerning fixed-point free actions of finite groups on finite, contractible complexes which is required for the discussion in Section 3. 
The author expects that the results proved in this article will also have applications in the algebraic $K$-theory of spaces; these will be presented elsewhere.

Acknowledgements This article contains parts of the author's $\mathrm{PhD}$ thesis written under the supervision of Arthur Bartels, whose support and advice are gratefully acknowledged. The author would like to thank the referee for suggesting the present form of Definition 2.1. Daniel Kasprowski provided helpful comments on an earlier version of this article. The author was financially supported by CRC 878 Groups, Geometry and Actions of the German Research Foundation (DFG).

\section{Resolving fixed points of group actions on simplicial complexes}

Our strategy is not to come up with entirely new proofs whenever we wish to replace an invocation of the Farrell-Hsiang method. Instead, we will relate (a variant of) the Farrell-Hsiang condition to the notion of transfer reducibility, and then improve the existing verifications of the Farrell-Hsiang condition.

The major difference between the proofs of the Farrell-Jones conjecture in Bartels, Lück and Reich [7] and Bartels and Lück [5] lies in the construction of the transfer maps. While the proof in [7] exploits the existence of a compact transfer space, the Farrell-Hsiang method relies on a discrete $G$-set for the transfer and uses an algebraic result due to Swan [27, Corollary 4.2(c) and Proposition 1.1] as additional input.

In this section, we prove a result on the space level which is analogous to Swan's induction theorem. This will enable us to produce appropriate transfer spaces.

The objects of interest are $G$-simplicial complexs; these are (abstract) simplicial complexes $X$ equipped with a $G$-action such that whenever a group element $g$ fixes a simplex $x=\left\{x_{0}, \ldots, x_{n}\right\}$ in $X$, then $g x_{i}=x_{i}$ for all $0 \leq i \leq n$. Note that for such a complex, the entire group action is encoded in the action of $G$ on the set of 0 -simplices $X_{0}$. The geometric realization of a $G$-simplicial complex is a $G-\mathrm{CW}$-complex.

2.1 Definition Let $X$ be a $G$-simplicial complex. A set of resolution data $\left(\mathcal{R}, \rho_{0}\right)$ for $X$ is a $G$-simplicial complex over $X_{0}$, ie a map of $G$-simplicial complexes $\rho_{0}: \mathcal{R} \rightarrow X_{0}$, where we regard the set of vertices $X_{0}$ as a 0 -dimensional $G$-simplicial complex.

If $\left(\mathcal{R}, \rho_{0}\right)$ is a set of resolution data for some $G$-simplicial complex $X$, the fiber $\rho_{0}^{-1}(x)$ of $\rho_{0}$ is a $G_{x}$-simplicial complex for each $x \in X_{0}$. We denote this complex by $\mathcal{R}(x)$. 
2.2 Definition Let $X$ be a $G$-simplicial complex and $\left(\mathcal{R}, \rho_{0}\right)$ be a set of resolution data for $X$. We define a simplicial complex $X\left[\mathcal{R}, \rho_{0}\right]$ as follows: The set of vertices is given by $\mathcal{R}_{0}$. A set $y=\left\{y_{0}, \ldots, y_{n}\right\}$ spans an $n$-simplex in $X\left[\mathcal{R}, \rho_{0}\right]$ if the following holds:

- For every $x \in X_{0}$, the set $y \cap \mathcal{R}(x)_{0}$ is either empty or a simplex in $\mathcal{R}(x)$.

- The set $\rho_{0}(y)$ is a simplex in $X$.

The group $G$ acts on $X\left[\mathcal{R}, \rho_{0}\right]$ by

$$
g \cdot\left\{y_{0}, \ldots, y_{n}\right\}:=\left\{g y_{0}, \ldots, g y_{n}\right\} .
$$

The map $\rho_{0}$ induces a map of simplicial complexes

$$
\rho: X\left[\mathcal{R}, \rho_{0}\right] \rightarrow X, \quad y \mapsto \rho_{0}(y),
$$

which we call the resolution of $X$ by $\left(\mathcal{R}, \rho_{0}\right)$.

It is easy to check that $X\left[\mathcal{R}, \rho_{0}\right]$ is indeed a $G$-simplicial complex and that $\rho$ is a map of $G$-simplicial complexes. Let us observe that any simplex $y \in X\left[\mathcal{R}, \rho_{0}\right]$ can be partitioned into

$$
y=\coprod_{x \in \rho_{0}(y)} y \cap \mathcal{R}(x)_{0} .
$$

In particular, a subgroup of $G$ can only appear as a stabilizer of $X\left[\mathcal{R}, \rho_{0}\right]$ if it is a stabilizer group of some $\mathcal{R}(x)$.

If $X$ is $n$-dimensional and there is some $k$ such that the dimension of $\mathcal{R}(x)$ is at most $k$ for all $x$, it follows that the dimension of $X\left[\mathcal{R}, \rho_{0}\right]$ can be bounded by $(n+1)(k+1)-1=n k+n+k$.

A map of sets of resolution data is a map $\tau_{0}:\left(\mathcal{R}, \rho_{0}\right) \rightarrow\left(\mathcal{R}^{\prime}, \rho_{0}^{\prime}\right)$ of $G$-simplicial complexes over $X_{0}$, ie a map of $G$-simplicial complexes $\tau: \mathcal{R} \rightarrow \mathcal{R}^{\prime}$ such that $\rho_{0}=$ $\rho_{0}^{\prime} \circ \tau_{0}$. Note that such a map necessarily restricts to a map of $G_{x}$-simplicial complexes $\tau_{0}(x): \mathcal{R}(x) \rightarrow \mathcal{R}^{\prime}(x)$ for each $x \in X_{0}$. Every map of sets of resolution data $\tau_{0}$ induces a canonical map $\tau:\left(X\left[\mathcal{R}, \rho_{0}\right], \rho\right) \rightarrow\left(X\left[\mathcal{R}^{\prime}, \rho_{0}^{\prime}\right], \rho^{\prime}\right)$ of $G$-simplicial complexes over $X$.

2.3 Remark Let $X$ be a $G$-simplicial complex and let $\left(\mathcal{R}, \rho_{0}\right)$ be a set of resolution data for $X$. Define $\left|X, \mathcal{R}, \rho_{0}\right|$ as the set of formal finite convex combinations $\left|X, \mathcal{R}, \rho_{0}\right|:=\left\{\sum_{x \in X_{0}} \lambda_{x} \cdot \eta_{x}\left|\lambda_{x} \geq 0,\left\{x \mid \lambda_{x} \neq 0\right\} \in X, \sum_{x \in X_{0}} \lambda_{x}=1, \eta_{x} \in\right| \mathcal{R}(x) \mid\right\}$. 
Define

$$
F:\left|X\left[\mathcal{R}, \rho_{0}\right]\right| \rightarrow\left|X, \mathcal{R}, \rho_{0}\right|, \quad \sum_{y \in \mathcal{R}_{0}} \lambda_{y} \cdot y \mapsto \sum_{x \in X_{0}} \lambda_{x} \cdot\left(\sum_{y \in \rho_{0}^{-1}(x)} \frac{\lambda_{y}}{\lambda_{x}} \cdot y\right),
$$

where $\lambda_{x}:=\sum_{y \in \rho_{0}^{-1}(x)} \lambda_{y}$. Since we think of points in $\left|X, \mathcal{R}, \rho_{0}\right|$ as finite sums by ignoring all terms whose coefficient is zero, we do not worry about the fact that $\lambda_{y} / \lambda_{x}$ is undefined when $\lambda_{x}=0$.

Write $\eta_{x}=\sum_{y \in \mathcal{R}(x)_{0}} \eta_{x, y} \cdot y$. Then we can also define

$$
F^{\prime}:\left|X, \mathcal{R}, \rho_{0}\right| \rightarrow\left|X\left[\mathcal{R}, \rho_{0}\right]\right|, \quad \sum_{x \in X_{0}} \lambda_{x} \cdot \eta_{x} \mapsto \sum_{y \in \mathcal{R}_{0}} \lambda_{\rho_{0}(y)} \eta_{\rho_{0}(y), y} \cdot y .
$$

Then $F$ and $F^{\prime}$ are mutually inverse bijections. This will turn out to be a more convenient model for $\left|X\left[\mathcal{R}, \rho_{0}\right]\right|$. In particular, the $\ell^{1}$-metric on $\left|X\left[\mathcal{R}, \rho_{0}\right]\right|$ induces a metric $d^{1}$ on $\left|X, \mathcal{R}, \rho_{0}\right|$ via $F^{\prime}$.

2.4 Proposition Let $X$ be a $G$-simplicial complex and let $\left(\mathcal{R}, \rho_{0}\right)$ and $\left(\mathcal{R}^{\prime}, \rho_{0}^{\prime}\right)$ be sets of resolution data for $X$. Suppose that $\tau_{0}:\left(\mathcal{R}, \rho_{0}\right) \rightarrow\left(\mathcal{R}^{\prime}, \rho_{0}^{\prime}\right)$ is a map of sets of resolution data such that the restriction $\tau_{0}(x): \mathcal{R}(x) \rightarrow \mathcal{R}^{\prime}(x)$ is a homotopy equivalence (in the nonequivariant sense) for all $x \in X_{0}$.

Then the induced map $\tau: X\left[\mathcal{R}, \rho_{0}\right] \rightarrow X\left[\mathcal{R}^{\prime}, \rho_{0}^{\prime}\right]$ is a homotopy equivalence in the nonequivariant sense.

Proof Choose a homotopy inverse $f_{x}:\left|\mathcal{R}^{\prime}(x)\right| \rightarrow|\mathcal{R}(x)|$ to $\left|\tau_{0}(x)\right|$ for every $x \in X_{0}$, and let $H_{x}:|\mathcal{R}(x)| \times[0,1] \rightarrow|\mathcal{R}(x)|$ and $H_{x}^{\prime}:\left|\mathcal{R}^{\prime}(x)\right| \times[0,1] \rightarrow\left|\mathcal{R}^{\prime}(x)\right|$ be homotopies witnessing $f_{x} \circ\left|\tau_{0}(x)\right| \simeq \operatorname{id}_{|\mathcal{R}(x)|}$ and $\left|\tau_{0}(x)\right| \circ f_{x} \simeq \mathrm{id}_{\left|\mathcal{R}^{\prime}(x)\right|}$, respectively.

Using the alternative description of $\left|X\left[\mathcal{R}, \rho_{0}\right]\right|$ from Remark 2.3, we can define a (nonequivariant) map

$$
f:\left|X, \mathcal{R}^{\prime}, \rho_{0}^{\prime}\right| \rightarrow\left|X, \mathcal{R}, \rho_{0}\right|, \quad \sum_{x} \lambda_{x} \cdot \eta_{x} \mapsto \sum_{x} \lambda_{x} \cdot f_{x}\left(\eta_{x}\right) .
$$

Similarly, there are induced homotopies $H:\left|X, \mathcal{R}, \rho_{0}\right| \times[0,1] \rightarrow\left|X, \mathcal{R}, \rho_{0}\right|$ and $H^{\prime}:\left|X, \mathcal{R}^{\prime}, \rho_{0}^{\prime}\right| \times[0,1] \rightarrow\left|X, \mathcal{R}^{\prime}, \rho_{0}^{\prime}\right|$, and it is easy to check that these witness $f \circ|\tau| \simeq \operatorname{id}_{\left|X, \mathcal{R}, \rho_{0}\right|}$ and $|\tau| \circ f \simeq \operatorname{id}_{\left|X, \mathcal{R}^{\prime}, \rho_{0}^{\prime}\right|}$, respectively.

2.5 Corollary Let $X$ be a $G$-simplicial complex and $\left(\mathcal{R}, \rho_{0}\right)$ be a set of resolution data on $X$. Suppose that $\mathcal{R}(x)$ is contractible for all $x \in X_{0}$. Then the resolution $X\left[\mathcal{R}, \rho_{0}\right] \rightarrow X$ is a (nonequivariant) homotopy equivalence. 
We can use this construction to reduce the size of the stabilizers of a $G$-simplicial complex incrementally. Specifically, we will give an answer to the question of what the smallest possible stabilizers of a finite group action on a finite, contractible complex are.

2.6 Definition Let $\mathcal{C} y c$ denote the family of finite cyclic groups. For a given prime $p$, we let

$$
\mathcal{C} y c_{p}:=\left\{\begin{array}{l|l}
H & \begin{array}{l}
\text { There is an extension } 1 \rightarrow P \rightarrow H \rightarrow C \rightarrow 1 \\
\text { such that } P \text { is a finite } p \text {-group and } C \in \mathcal{C} y c .
\end{array}
\end{array}\right\}
$$

denote the class of groups which are cyclic $\bmod p$.

Finally, we call

$$
\mathcal{D} r:=\left\{\begin{array}{l|l}
G & \begin{array}{l}
\text { There is an extension } 1 \rightarrow H \rightarrow G \rightarrow Q \rightarrow 1 \text { such that } H \in \mathcal{C} y c_{p} \\
\text { and } Q \text { is a finite } q \text {-group for some primes } p \text { and } q .
\end{array}
\end{array}\right\}
$$

the Dress family.

2.7 Remark Recall that a finite group $G$ is hyperelementary if there is an extension $1 \rightarrow C \rightarrow G \rightarrow Q \rightarrow 1$ such that $C$ is cyclic and $Q$ is a $q$-group for some prime $q$. Hence, every finite hyperelementary group is also a Dress group.

2.8 Definition Let $G$ be a finite group. Define the depth of $G$ to be

$$
d(G):=\sup \left\{\begin{array}{l|l}
n & \begin{array}{l}
\text { There is a properly descending chain of subgroups } \\
G_{1} \supsetneqq G_{2} \supsetneqq \cdots \supsetneq G_{n} \text { in } G .
\end{array}
\end{array}\right\} .
$$

Observe that it is easy to find upper bounds for the depth of a finite group. If $|G|=$ $p_{1}^{k_{1}} \cdots p_{r}^{k_{r}}$ is the prime factorization of the order of $G$, the depth of $G$ cannot exceed $k_{1}+\cdots+k_{r}+1$.

2.9 Theorem (Oliver) There exists a monotonely increasing, affine linear function bd: $\mathbb{N}_{+} \rightarrow \mathbb{N}_{+}$such that for every finite group $G \notin \mathcal{D r}$, there is a finite, contractible $G$-simplicial complex $X$ with $X^{G}=\varnothing$ whose dimension is bounded by bd $(d(G))$.

Proof Excluding the dimension bound, this is stated as one of the main results of [19]. Nevertheless, the proof given by Oliver can be seen to provide the claimed bound. A short review of the proof which makes this explicit can be found in the appendix.

2.10 Corollary For every finite group $G$, there is a finite, contractible $G$-simplicial complex $X$ whose stabilizers lie in $\mathcal{D r}$ and whose dimension is bounded by

$$
\sum_{\varnothing \neq M \subset\{1, \ldots, d(G)\}} \prod_{m \in M} \operatorname{bd}(m) \leq 2^{d(G)} \cdot \operatorname{bd}(d(G))^{d(G)} .
$$


Proof Define for any positive natural number $d$

$$
\delta(d):=\sum_{\varnothing \neq M \subset\{1, \ldots, d\}} \prod_{m \in M} \operatorname{bd}(m) .
$$

If $d(G)=1$, there is nothing to show. So write $d(G)=d+1>1$. We can assume without loss of generality that $G \notin \mathcal{D} r$. Theorem 2.9 asserts the existence of a finite, contractible $G$-simplicial complex $X^{\prime}$ which does not have a global fixed point and whose dimension is bounded by $\operatorname{bd}(d(G))$. Picking a set of representatives $\left\{x_{i}\right\}_{i \in I}$ for the $G$-orbits in $X_{0}^{\prime}$ fixes a $G$-equivariant bijection $X_{0}^{\prime} \cong \bigsqcup_{i \in I} G / G_{x_{i}}$, where each $G_{x_{i}}$ is a proper subgroup of $G$. For each $i \in I$, there is by induction a finite, contractible $G_{x_{i}}$-simplicial complex $\mathcal{R}\left(x_{i}\right)$ whose stabilizers lie in $\mathcal{D r}$ and whose dimension is bounded by $\delta(d)$. Then the projection maps pr: $\mathcal{R}\left(x_{i}\right) \rightarrow G_{x_{i}} / G_{x_{i}}$ induce a set of resolution data

$\rho_{0}: \mathcal{R}=\coprod_{i \in I} G \times_{G_{x_{i}}} \mathcal{R}\left(x_{i}\right) \stackrel{\bigsqcup_{i \in I} G \times_{G_{x_{i}}} \mathrm{pr}}{\longrightarrow} \coprod_{i \in I} G \times_{G_{x_{i}}} G_{x_{i}} / G_{x_{i}} \cong \coprod_{i \in I} G / G_{x_{i}} \cong X_{0}^{\prime}$.

Then $X:=X^{\prime}\left[\mathcal{R}, \rho_{0}\right]$ is a finite $G$-simplicial complex whose stabilizers lie in $\mathcal{D} r$. It is contractible by Corollary 2.5. As observed earlier, its dimension is bounded by

$$
\begin{aligned}
& \mathrm{bd}(d+1) \cdot \delta(d)+\operatorname{bd}(d+1)+\delta(d) \\
& =\operatorname{bd}(d+1) \cdot\left(\sum_{\varnothing \neq M \subset\{1, \ldots, d\}} \prod_{m \in M} \mathrm{bd}(m)\right)+\operatorname{bd}(d+1)+\delta(d) \\
& =\sum_{\varnothing \neq M \subset\{1, \ldots, d\}}\left(\mathrm{bd}(d+1) \cdot \prod_{m \in M} \mathrm{bd}(m)\right)+\mathrm{bd}(d+1)+\delta(d)
\end{aligned}
$$

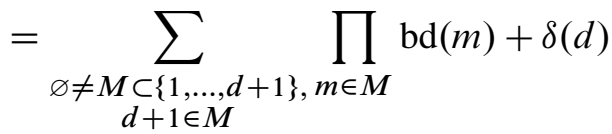

$$
\begin{aligned}
& =\sum_{\varnothing \neq M \subset\{1, \ldots, d+1\},} \prod_{\substack{d+1 \in M \\
d \in M}} \mathrm{bd}(m)+\sum_{\substack{\varnothing \neq M \subset\{1, \ldots, d+1\} \\
d+1 \notin M}} \prod_{m \in M} \mathrm{bd}(m) \\
& =\sum_{\varnothing \neq M \subset\{1, \ldots, d+1\}} \prod_{m \in M} \operatorname{bd}(m)=\delta(d+1) .
\end{aligned}
$$

The weaker bound can be obtained from this by estimating $m \leq d(G)$ and $|M| \leq d(G)$.

The $G$-simplicial complex $X$ from Corollary 2.10 will typically not be a model for $E_{\mathcal{D r}} G$. See Oliver [20, bottom of page 93] for a proof of this. 


\section{A variant of the Farrell-Hsiang condition}

We are now ready to formulate our strengthening of the Farrell-Hsiang condition and to relate it to the property of being transfer reducible. Whenever we speak about generating sets of groups, we assume these to be symmetric for convenience.

3.1 Definition Let $G$ be a group and $S$ a finite generating set for $G$. Let $\mathcal{F}$ be a family of subgroups of $G$.

Call $(G, S)$ a Dress-Farrell-Hsiang group of bounded depth with respect to $\mathcal{F}$ if there exist $N \in \mathbb{N}$ and $B \in \mathbb{N}$ such that for every $\varepsilon>0$ there are:

- An epimorphism $\pi: G \rightarrow F$ to a finite group with depth $d(F) \leq B$.

- For every subgroup $D \leq F$ with $D \in \mathcal{D r}$ a $G$-simplicial complex $E_{D}$ of dimension at most $N$ whose isotropy groups lie in $\mathcal{F}$, and a $\bar{D}:=\pi^{-1}(D)$ equivariant map $f_{D}: G \rightarrow E_{D}$ such that $d^{\ell^{1}}\left(f_{D}(g), f_{D}\left(g^{\prime}\right)\right) \leq \varepsilon$ whenever $g^{-1} g^{\prime} \in S$.

3.2 Definition We say that $(G, S)$ is combinatorially transfer reducible with respect to $\mathcal{F}$ if there exists $v \in \mathbb{N}$ such that for every $\varepsilon>0$ there are:

- A finite, contractible $G$-simplicial complex $X$ of dimension at most $v$.

- A $G$-simplicial complex $E$ of dimension at most $v$ whose isotropy groups lie in $\mathcal{F}$.

- A map $f: X \rightarrow E$ which is $S$-equivariant up to $\varepsilon$, ie such that

$$
d^{\ell^{1}}(s f(x), f(s x)) \leq \varepsilon
$$

holds for all $s \in S$ and $x \in X$.

The Farrell-Hsiang condition of Bartels and Lück [5, Definition 1.1; 2, Definition 2.14] and Bartels [1, Theorem C] can be obtained from Definition 3.1 by dropping the assumption on $d(F)$ and replacing the Dress family by the family of hyperelementary subgroups of $F$. Hence, being a Dress-Farrell-Hsiang group of bounded depth implies being a Farrell-Hsiang group (see Remark 2.7). Similarly, combinatorial transfer reducibility is a very special case of the more general notions of transfer reducibility explained in Bartels [1, Theorem A and Theorem B] (see also Bartels and Lück [4, Definition 1.8; 6, Definition 0.4]).

It was shown by Bartels and Lück [5] that every Farrell-Hsiang group satisfies the Farrell-Jones conjectures in algebraic $K$ - and $L$-theory. The validity of the FarrellJones conjecture for transfer reducible groups was established in Bartels, Lück and 
Reich [7] (for algebraic $K$-theory) and Bartels and Lück [4] (for $L$-theory); see [1, Theorem A]. Hence, the Farrell-Jones conjecture is known to hold both for DressFarrell-Hsiang groups and for combinatorially transfer reducible groups. As explained in the introduction, the purpose of Theorem 3.4 below is to point out an alternative proof of the conjecture for Dress-Farrell-Hsiang groups which uses only the results of [7] and [4] instead of [5].

An immediate consequence of Corollary 2.10 is the following variant of the result that finite groups satisfy the isomorphism conjecture with respect to the family of hyperelementary subgroups [3, Theorem 2.9 and Lemma 4.1]:

3.3 Corollary Every finite group is combinatorially transfer reducible with respect to $\mathcal{D} r$. In particular, the assembly map

$$
\alpha_{\mathcal{D} r}^{G}: H_{n}^{G}\left(E_{\mathcal{D} r} G ; \mathbb{K}_{\mathcal{A}}^{-\infty}\right) \rightarrow K_{n}\left(\mathcal{A} *_{G} G / G\right)
$$

is an isomorphism for every finite group $G$ and every small additive $G$-category $\mathcal{A}$.

Proof Take $X$ as in Corollary 2.10 and $f=\mathrm{id}_{X}$.

The more important observation is the following.

3.4 Theorem Let $G$ be a group and $S$ a finite generating set for $G$. Let $\mathcal{F}$ be a family of subgroups of $G$. If $(G, S)$ is a Dress-Farrell-Hsiang group of bounded depth with respect to $\mathcal{F}$, then it is combinatorially transfer reducible with respect to $\mathcal{F}$.

Proof Let $N$ and $B$ be the natural numbers whose existence is asserted in the definition of a Dress-Farrell-Hsiang group of bounded depth. Fix $\varepsilon>0$, and pick an appropriate epimorphism $\pi: G \rightarrow F$ to a finite group with $d(F) \leq B$. By Corollary 2.10, there exists a finite, contractible $F$-simplicial complex $X$ whose dimension is bounded by $\beta:=2^{B} \cdot \operatorname{bd}(B)^{B}$ (note that this bound only depends on $B$ ) and whose isotropy groups lie in $\mathcal{D r}$. We equip $X$ with a $G$-action by restricting the $F$-action along $\pi$.

For the remainder of the proof, fix a representative of each $G$-orbit in $X_{0}$. These choices induce a $G$-equivariant bijection

$$
h_{0}: \coprod_{i \in I} G / \bar{D}_{i} \cong X_{0},
$$

where for each $i$, the subgroup $\bar{D}_{i}$ is the preimage under $\pi$ of some $D_{i} \leq F$ with $D_{i} \in \mathcal{D} r$. The bijection $h_{0}$ allows us to define a $G$-simplicial complex $Y$ with $Y_{0}=\bigsqcup_{i \in I} G / \bar{D}_{i}$ and a $G$-equivariant isomorphism $h: Y \rightarrow X$ which restricts to $h_{0}$ on the vertex sets. 
Now use the Dress-Farrell-Hsiang assumption to pick for each $i \in I$ a $G$-simplicial complex $E_{i}^{\prime}$ whose dimension is at most $N$ and whose isotropy groups lie in $\mathcal{F}$, as well as a $\bar{D}_{i}$-equivariant map $f_{i}^{\prime}: G \rightarrow E_{i}^{\prime}$ such that $d^{\ell^{1}}\left(f_{i}^{\prime}(g), f_{i}^{\prime}\left(g^{\prime}\right)\right) \leq \varepsilon$ whenever $g^{-1} g^{\prime} \in S$. Define $E_{i}:=G \times \bar{D}_{i} E_{i}^{\prime}$ and set $\mathcal{E}:=\bigsqcup_{i \in I} E_{i}$. Let $\eta_{0}$ denote the composition

$$
\mathcal{E}=\coprod_{i \in I} G \times \bar{D}_{i} E_{i}^{\prime} \stackrel{\amalg_{i} G \times \bar{D}_{i} \mathrm{pr}}{\longrightarrow} \coprod_{i \in I} G \times \bar{D}_{i} \bar{D}_{i} / \bar{D}_{i} \cong \coprod_{i \in I} G / \bar{D}_{i}=Y_{0},
$$

where pr: $E_{i}^{\prime} \rightarrow \bar{D}_{i} / \bar{D}_{i}$ is the obvious projection map. Then $\eta_{0}$ is $G$-equivariant, so we have defined a set of resolution data $\left(\mathcal{E}, \eta_{0}\right)$ for $Y$. Consider the resolution $\eta: Y\left[\mathcal{E}, \eta_{0}\right] \rightarrow Y$. All isotropy groups of the $G$-simplicial complex $Y\left[\mathcal{E}, \eta_{0}\right]$ lie in $\mathcal{F}$, and its dimension is bounded by $v:=\beta N+\beta+N$. Observe that $v$ depends only on $N$ and $B$.

We claim that there is a section $f:|Y| \rightarrow\left|Y\left[\mathcal{E}, \eta_{0}\right]\right|$ of $|\eta|$ which is $S$-equivariant up to $\varepsilon$; for our argument, it is irrelevant that $f$ is a section to $\eta$, but the $f$ we define will have this property. Once we have shown this, $f \circ|h|^{-1}$ is the map we require to finish the proof.

Define

$$
f_{i}: G / \bar{D}_{i} \rightarrow E_{i}, \quad g \bar{D}_{i} \mapsto\left(g, f_{i}^{\prime}\left(g^{-1}\right)\right) .
$$

Observe that every point in $|Y|$ can be written as

$$
\sum_{y \in Y_{0}} \lambda_{y} \cdot y=\sum_{i \in I} \sum_{g \bar{D}_{i} \in G / \bar{D}_{i}} \lambda_{g \bar{D}_{i}} \cdot g \bar{D}_{i}
$$

for appropriate $\lambda_{g \bar{D}_{i}}$. Hence, we can define a map $\widehat{f}:|Y| \rightarrow\left|Y, \mathcal{E}, \eta_{0}\right|$ by

$$
\widehat{f}\left(\sum_{i \in I} \sum_{g \bar{D}_{i} \in G / \bar{D}_{i}} \lambda_{g \bar{D}_{i}} g \bar{D}_{i}\right):=\sum_{i \in I} \sum_{g \bar{D}_{i} \in G / \bar{D}_{i}} \lambda_{g \bar{D}_{i}} f_{i}\left(g \bar{D}_{i}\right) .
$$

This is well-defined because $\eta\left(f_{i}\left(g \bar{D}_{i}\right)\right)=\eta\left(g, f_{i}^{\prime}\left(g^{-1}\right)\right)=g \bar{D}_{i}$. Set $f:=F^{\prime} \circ \widehat{f}$, where $F^{\prime}$ is the bijection

$$
\left|Y, \mathcal{E}, \eta_{0}\right| \stackrel{\cong}{\longrightarrow}\left|Y\left[\mathcal{E}, \eta_{0}\right]\right|
$$

from Remark 2.3.

Let $\zeta=\sum_{y} \lambda_{y} \cdot \zeta_{y}$ and $\vartheta=\sum_{y} \mu_{y} \cdot \vartheta_{y}$ be arbitrary points in $\left|Y, \mathcal{E}, \eta_{0}\right|$. Then we have

$$
d^{\ell^{1}}\left(F^{\prime}(\zeta), F^{\prime}(\vartheta)\right)=\sum_{e \in \mathcal{E}_{0}}\left|\lambda_{\eta_{0}(e)} \zeta_{\eta_{0}(e), e}-\mu_{\eta_{0}(e)} \vartheta_{\eta_{0}(e), e}\right|
$$


Using the triangle inequality, we can bound this by

$$
\begin{aligned}
\sum_{e \in \mathcal{E}_{0}} \mid & \lambda_{\eta_{0}(e)} \zeta_{\eta_{0}(e), e}-\mu_{\eta_{0}(e)} \vartheta_{\eta_{0}(e), e} \mid \\
& \leq \sum_{e \in \mathcal{E}_{0}}\left|\lambda_{\eta_{0}(e)} \zeta_{\eta_{0}(e), e}-\lambda_{\eta_{0}(e)} \vartheta_{\eta_{0}(e), e}\right|+\sum_{e \in \mathcal{E}_{0}}\left|\lambda_{\eta_{0}(e)} \vartheta_{\eta_{0}(e), e}-\mu_{\eta_{0}(e)} \vartheta_{\eta_{0}(e), e}\right| \\
& =\sum_{y \in Y_{0}}\left(\lambda_{y} \cdot \sum_{e \in \mathcal{E}(y)_{0}}\left|\zeta_{y, e}-\vartheta_{y, e}\right|\right)+\sum_{y \in Y_{0}}\left(\left|\lambda_{y}-\mu_{y}\right| \cdot \sum_{e \in \mathcal{E}(y)_{0}} \vartheta_{y, e}\right) \\
& =\sum_{y \in Y_{0}} \lambda_{y} \cdot d^{\ell^{1}}\left(\zeta_{y}, \vartheta_{y}\right)+\sum_{y \in Y_{0}}\left|\lambda_{y}-\mu_{y}\right| .
\end{aligned}
$$

We will now use this estimate to prove that the map $f$ is $S$-equivariant up to $\varepsilon$. Let $s \in S$. Then we have

$$
\begin{aligned}
d^{\ell^{1}}(s \cdot f & \left.\left(\sum_{i \in I} \sum_{g \bar{D}_{i} \in G / \bar{D}_{i}} \lambda_{g \bar{D}_{i}} g \bar{D}_{i}\right), f\left(s \cdot \sum_{i \in I} \sum_{g \bar{D}_{i} \in G / \bar{D}_{i}} \lambda_{g \bar{D}_{i}} g \bar{D}_{i}\right)\right) \\
= & d^{\ell^{1}}\left(F^{\prime}\left(\sum_{i \in I} \sum_{g \bar{D}_{i} \in G / \bar{D}_{i}} \lambda_{s^{-1} g \bar{D}_{i}}\left(g, f_{i}^{\prime}\left(g^{-1} s\right)\right)\right),\right. \\
& \left.F^{\prime}\left(\sum_{i \in I} \sum_{g \bar{D}_{i} \in G / \bar{D}_{i}} \lambda_{s^{-1} g \bar{D}_{i}}\left(g, f_{i}^{\prime}\left(g^{-1}\right)\right)\right)\right) \\
& \leq \sum_{i \in I} \sum_{g \bar{D}_{i} \in G / \bar{D}_{i}} \lambda_{s^{-1} g \bar{D}_{i}} d^{\ell^{1}}\left(\left(g, f_{i}^{\prime}\left(g^{-1} s\right)\right),\left(g, f_{i}^{\prime}\left(g^{-1}\right)\right)\right) \\
& +\sum_{i \in I} \sum_{g \bar{D}_{i} \in G / \bar{D}_{i}}\left|\lambda_{s^{-1} g \bar{D}_{i}}-\lambda_{s^{-1} g \bar{D}_{i}}\right|
\end{aligned}
$$

since the $\ell^{1}$-metric is $G$-invariant and $s^{-1} g g^{-1}=s^{-1} \in S$. So $f$ is $S$-equivariant up to $\varepsilon$, and the same is true about $f \circ|h|^{-1}$ since $h$ is an isomorphism of $G$-simplicial complexes.

\section{Overview of the Bartels-Farrell-Lück-Quinn argument}

To keep the exposition in this and the following two sections short, we do not elaborate on a number of arguments which are carried out in full detail in Bartels, Farrell and Lück [2]; the reader is advised to keep a well-read copy of [2] close-by.

We are now going to give an outline of the proof of Theorem 1.1 in [2]. There are two classes of groups which play a particularly prominent role: 
A group $\Gamma$ is called crystallographic if it contains a normal subgroup $A$ which is finitely generated and free abelian such that $A$ has finite index and equals its own centralizer in $\Gamma$. The subgroup $A$ is unique; the rank of $\Gamma$ is defined to be the rank of $A$, and equals the virtual cohomological dimension of $\Gamma$ (see [2, Section 3.1]).

The second important class of groups is that of special affine groups; these are those groups $\Gamma$ for which there is an extension

$$
1 \rightarrow \Theta \rightarrow \Gamma \rightarrow \Delta \rightarrow 1
$$

and an action $\rho^{\prime}: \Gamma \times \mathbb{R}^{n} \rightarrow \mathbb{R}^{n}$ by affine motions such that the restriction of $\rho^{\prime}$ to $\Theta$ is a cocompact, isometric and proper action, and $\Delta$ is either infinite cyclic or infinite dihedral. A special affine group $\Gamma$ is irreducible if for every epimorphism $\Gamma \rightarrow \Gamma^{\prime}$ onto a virtually finitely generated abelian group $\Gamma^{\prime}$, the virtual cohomological dimension of $\Gamma^{\prime}$ is at most 1 .

The proof of the Farrell-Jones conjecture for virtually poly- $\mathbb{Z}$-groups in [2] makes heavy use of a number of inheritance properties of the conjecture. In order to keep the exposition short, we do not recall these here, but refer the reader instead to [2, Section 2.3] for a quick overview.

Let $G$ be a virtually poly- $\mathbb{Z}$-group. Using the transitivity principle, the proof can proceed by induction on the virtual cohomological dimension of $G$. One may assume that $\operatorname{vcd}(G) \geq 2$. Then there is an extension

$$
1 \rightarrow G_{0} \rightarrow G \stackrel{\mathrm{pr}}{\rightarrow} \Gamma \rightarrow 1
$$

in which $G_{0}$ is either finite or a virtually poly- $\mathbb{Z}$-group which satisfies $\operatorname{vcd}\left(G_{0}\right) \leq$ $\operatorname{vcd}(G)-2$, and $\Gamma$ is a crystallographic or a special affine group. If $V$ is a virtually cyclic subgroup of $\Gamma$, its preimage under pr is a virtually poly- $\mathbb{Z}$-group with $\operatorname{vcd}\left(\operatorname{pr}^{-1}(V)\right)<\operatorname{vcd}(G)$. So one only needs to prove the conjecture for $\Gamma$.

4.1 Claim Every crystallographic group satisfies the Farrell-Jones conjecture.

4.2 Claim Every irreducible special affine group satisfies the Farrell-Jones conjecture.

Assuming the two claims, we need only consider the case that $\Gamma$ is a special affine group which is not irreducible. Pick an epimorphism $p: \Gamma \rightarrow \Gamma^{\prime}$, where $\Gamma^{\prime}$ is some virtually finitely generated abelian group with $\operatorname{vcd}\left(\Gamma^{\prime}\right) \geq 2$. For any virtually cyclic subgroup $V$ of $\Gamma^{\prime}$, the preimage $p^{-1}(V)$ is a virtually poly- $\mathbb{Z}-\operatorname{group}$ with $\operatorname{vcd}\left(p^{-1}(V)\right)<\operatorname{vcd}(G)$. This leaves the final case: 
4.3 Claim Every virtually finitely generated abelian group satisfies the Farrell-Jones conjecture.

In fact, the three claims also rely on each other: The proof of Claim 4.1 and Claim 4.3 is dealt with simultaneously as follows (see also Quinn [23], especially Proposition 2.4.1). One proceeds by induction over the virtual cohomological dimension of a virtually finitely generated abelian group $\Gamma$ as well as the smallest order of a finite group $F$ which fits into an exact sequence $1 \rightarrow \mathbb{Z}^{\operatorname{vcd}(\Gamma)} \rightarrow \Gamma \rightarrow F \rightarrow 1$; call the order of the group $F$ the holonomy of $\Gamma$.

One may assume that $\operatorname{vcd}(\Gamma) \geq 2$. Since there is an epimorphism with finite kernel onto a crystallographic group with the same virtual cohomological dimension, the Transitivity Principle serves to reduce the proof to the following claim:

4.4 Claim Every crystallographic group $\Gamma$ with $\operatorname{vcd}(\Gamma) \geq 2$ satisfies the isomorphism conjecture with respect to the family of all subgroups $G \leq \Gamma$ which satisfy one of the following conditions:

- $\operatorname{vcd}(G)<\operatorname{vcd}(\Gamma)$.

- $\operatorname{vcd}(G)=\operatorname{vcd}(\Gamma)$ and the holonomy of $G$ is smaller than the holonomy of $\Gamma$.

This takes care of Claim 4.1 and Claim 4.3. Once this has been done, the transitivity principle may be invoked another time to see that it suffices to show the following claim to finish the proof.

4.5 Claim Every irreducible special affine group satisfies the isomorphism conjecture with respect to the family of virtually finitely generated abelian groups.

The upshot of this discussion is that we will have to provide proofs for Claim 4.4 and Claim 4.5 which avoid the use of the Farrell-Hsiang method.

\section{Transfer reducibility of crystallographic groups}

Let us fix the following notational conventions: Recall that every crystallographic group $\Gamma$ fits into a short exact sequence

$$
1 \rightarrow A \rightarrow \Gamma \stackrel{\mathrm{pr}}{\rightarrow} F \rightarrow 1
$$

with $A$ a finitely generated, free abelian group which equals its own centralizer, and $F$ a finite group. Observe that there is always a canonical action of $F$ on $A$ by considering the conjugation actions of arbitrary lifts of elements of $F$ under pr. 
Let $s \in \mathbb{N}$. Then we denote by $A_{s}$ the quotient $A / s A$. Since $s A$ is also normal in $\Gamma$, we define $\Gamma_{s}$ to be the quotient $\Gamma / s A$. We let $\pi_{s}: \Gamma \rightarrow \Gamma_{s}$ be the projection map. Moreover, the epimorphism pr induces a surjective homomorphism $\mathrm{pr}_{s}: \Gamma_{S} \rightarrow F$ whose kernel is precisely $A_{s}$.

The normal subgroup $A$ is isomorphic to $\mathbb{Z}^{n}$, where $n$ is the rank of $\Gamma$. Consequently, $A_{s} \cong \mathbb{Z}^{n} / s \mathbb{Z}^{n} \cong(\mathbb{Z} / s)^{n}$. It follows that $\left|\Gamma_{s}\right|=\left|A_{s}\right| \cdot|F|=s^{n} \cdot|F|$. Therefore, it suffices to bound the number of prime factors in $s$ (counted with their multiplicities) if we want to bound the depth of the finite quotient $\Gamma_{s}$.

We will make regular use of the following observation:

5.1 Lemma Let $G$ be a finite group, and suppose there is a normal series $P^{\prime} \unlhd H^{\prime} \unlhd G$ such that $P^{\prime}$ is a $p$-group, $H^{\prime} / P^{\prime}$ is cyclic and $G / H^{\prime}$ is a $q$-group, ie $G$ lies in $\mathcal{D} r$. Then there is a normal series $P \unlhd H \unlhd G$ such that $P$ is a $p$-group, $H / P$ is cyclic, $G / H$ is a $q$-group and neither $p$ nor $q$ divide the order of $H / P$. Moreover, $H$ is isomorphic to a semidirect product $P \rtimes H / P$, the subgroup $P$ is normal in $G$ and $G$ is $q$-hyperelementary mod $p$.

Proof Let $\pi_{1}: H^{\prime} \rightarrow H^{\prime} / P^{\prime}$ be the projection. Since $H^{\prime} / P^{\prime}$ is cyclic, there is a unique cyclic $p$-Sylow group $S_{p}$ in $H^{\prime} / P^{\prime}$; so $H^{\prime} / P^{\prime}$ decomposes as a direct product $H^{\prime} / P^{\prime} \cong S_{p} \times C^{\prime}$. Set $P:=\pi_{1}^{-1}\left(S_{p}\right)$. Then $P$ is a normal $p$-group in $H^{\prime}$, and the quotient $H^{\prime} / P$ is isomorphic to $C^{\prime}$.

If $p=q$, we set $H:=H^{\prime}$ and are done. Otherwise, let $\pi_{2}: H^{\prime} \rightarrow H^{\prime} / P \cong C^{\prime}$ be the projection, and let $S_{q}$ be the unique $q$-Sylow group of $C^{\prime}$. We have an isomorphism $C^{\prime} \cong C \times S_{q}$. Set $H:=\pi_{2}^{-1}(C)$.

We claim that $H$ is normal in $G$. Let $h \in H, g \in G$, and suppose that $g h g^{-1} \notin H$. Since $H^{\prime}$ is normal in $G$, we know that $g h g^{-1} \in H^{\prime}$. The order of $g h g^{-1}$ equals the order of $h$, so we conclude from $p \neq q$ that no power of $q$ divides the order of $g h g^{-1}$. Since we assumed that $g h g^{-1} \notin H$, this element maps to a nontrivial element in $H^{\prime} / H \cong S_{q}$; but then there must be some power of $q$ which divides the order of $g h g^{-1}$, which is a contradiction.

Set $Q:=G / H$. The order of this group is given by

$$
|Q|=\frac{|G|}{|H|}=\frac{\left|H^{\prime}\right| \cdot\left|G / H^{\prime}\right|}{|H|}=\frac{|P| \cdot|C| \cdot\left|S_{q}\right| \cdot\left|G / H^{\prime}\right|}{|C| \cdot|P|}=\left|S_{q}\right| \cdot\left|G / H^{\prime}\right|,
$$

so $P \unlhd H \unlhd G$ is the desired normal series.

The Schur-Zassenhaus theorem [25, Theorem 9.3.6] states that $H$ is a semidirect product. While this also implies that $P$ is normal in $G$, we prove normality by hand. 
Let $x \in P$ and $g \in G$, and suppose that $g x g^{-1} \notin P$. Since $H$ is normal, we know that $g x g^{-1} \in H$. So $g x g^{-1}$ defines a nontrivial element in $H / P$. In particular, there is some prime $l \neq p$ which divides the order of $g_{x g^{-1}}$; this is a contradiction since $\left|g x g^{-1}\right|=|x|$ is a $p$-power. Moreover, the kernel of the natural surjection $G / P \rightarrow G / H$ is isomorphic to $H / P$, so $G / P$ is $q$-hyperelementary.

5.2 Lemma (cf Bartels-Farrell-Lück [2, Lemma 3.8]) The group $\mathbb{Z}^{2} \rtimes_{- \text {id }} \mathbb{Z} / 2$ is a Dress-Farrell-Hsiang group of bounded depth with respect to $\mathcal{V C} y c$ (relative to an arbitrary finite generating set).

Proof Set $\Gamma:=\mathbb{Z}^{2} \rtimes_{- \text {id }} \mathbb{Z} / 2$, and let

$$
1 \rightarrow \mathbb{Z}^{2} \stackrel{i}{\rightarrow} \Gamma \stackrel{\mathrm{pr}}{\rightarrow} \mathbb{Z} / 2 \rightarrow 1
$$

be the obvious short exact sequence. Let $d_{\Gamma}$ be the word metric with respect to some chosen finite generating set $S$ of $\Gamma$. The map ev: $\Gamma \rightarrow \mathbb{R}^{2}$ which evaluates the natural $\Gamma$-action on $\mathbb{R}^{2}$ at the point 0 is a quasi-isometry, so we can find positive constants $C_{1}$ and $C_{2}$ such that for all $\gamma_{1}, \gamma_{2} \in \Gamma$

$$
d_{\mathrm{euc}}\left(\operatorname{ev}\left(\gamma_{1}\right), \operatorname{ev}\left(\gamma_{2}\right)\right) \leq C_{1} \cdot d_{\Gamma}\left(\gamma_{1}, \gamma_{2}\right)+C_{2}
$$

holds. Let $\varepsilon>0$. Pick three pairwise distinct odd prime numbers $p_{1}, p_{2}, p_{3}$ which satisfy

$$
p_{i} \geq \frac{8 \cdot\left(C_{1}+C_{2}\right)^{2}}{\varepsilon^{2}} .
$$

Set $s:=p_{1} p_{2} p_{3}$, so $\Gamma_{s}:=(\mathbb{Z} / s \mathbb{Z})^{2} \rtimes_{-\mathrm{id}} \mathbb{Z} / 2$. This fits into an exact sequence

$$
1 \rightarrow(\mathbb{Z} / s)^{2} \rightarrow \Gamma_{s} \stackrel{\text { pr }_{s}}{\longrightarrow} \mathbb{Z} / 2 \rightarrow 1 .
$$

Since $\left|\Gamma_{s}\right|=2 \cdot\left(p_{1} p_{2} p_{3}\right)^{2}$, the depth of $\Gamma_{s}$ is uniformly bounded.

Let $D \leq \Gamma_{s}$ be a subgroup which lies in $\mathcal{D} r$. Then there is a normal series $Q_{0} \unlhd D_{0} \unlhd D$ such that $Q_{0}$ is a $q_{0}$-group, $D / D_{0}$ is a $q_{1}$-group, and $D_{0} / Q_{0}$ is a cyclic group of order prime to $q_{0}$ and $q_{1}$. Assume without loss of generality that $p_{3} \notin\left\{q_{0}, q_{1}\right\}$, and consider the projection $\pi: \Gamma_{s} \rightarrow \Gamma_{p_{3}}$. Then $\pi\left(D \cap(\mathbb{Z} / s)^{2}\right)$ is a cyclic subgroup of $\left(\mathbb{Z} / p_{3}\right)^{2} \leq \Gamma_{p_{3}}$.

If $\pi\left(D \cap(\mathbb{Z} / s)^{2}\right)$ is nontrivial, use [2, Lemma 3.7] to find a homomorphism $r: \mathbb{Z}^{2} \rightarrow$ $\mathbb{Z}$ such that the kernel of the mod $p_{3}$-reduction of $r$ equals $\pi\left(D \cap(\mathbb{Z} / s)^{2}\right)$, and $r_{\mathbb{R}}=r \otimes_{\mathbb{Z}} \mathbb{R}$ satisfies $d_{\text {euc }}\left(r_{\mathbb{R}}\left(x_{1}\right), r_{\mathbb{R}}\left(x_{2}\right)\right) \leq \sqrt{2 p_{3}} \cdot d\left(x_{1}, x_{2}\right)$ for all $x_{1}, x_{2} \in \mathbb{R}$. Otherwise, let $r: \mathbb{Z}^{2} \rightarrow \mathbb{Z}$ be the projection onto the first factor.

Set $\bar{D}:=\pi_{s}^{-1}(D)$. Then $r\left(\bar{D} \cap \mathbb{Z}^{2}\right) \subset p_{3} \mathbb{Z}$, and the argument proceeds precisely as in [2, Lemma 3.8] from here on. 
5.3 Proposition (cf Bartels-Farrell-Lück [2, Lemma 3.15]) Let $\Gamma$ be a crystallographic group of rank 2 which possesses a normal infinite cyclic subgroup. Let $\mathcal{F}$ be the family of subgroups of $\Gamma$ which contains all virtually cyclic groups as well as all groups which do not surject onto $F$ under pr.

Then $\Gamma$ is a Dress-Farrell-Hsiang group of bounded depth with respect to $\mathcal{F}$ (relative to an arbitrary finite generating set).

Proof We will have to use the following facts which are proved at the beginning of the proof of Lemma 3.15 in [2]:

- $A$ decomposes uniquely into a direct sum $Z_{1} \oplus Z_{2}$ of two infinite cyclic and $F$-invariant subgroups.

- $\quad F$ is either $\mathbb{Z} / 2$ or $\mathbb{Z} / 2 \times \mathbb{Z} / 2$.

If $Z$ is either $Z_{1}$ or $Z_{2}$, this is a normal subgroup of $\Gamma$ (since it is $F$-invariant). Let $\hat{\xi}_{Z}: \Gamma \rightarrow \Gamma / Z$ and $\xi_{Z}: A \rightarrow A / Z$ be the projection maps. Furthermore, there is an epimorphism $\widehat{\mu}_{Z}: \Gamma / Z \rightarrow \Delta_{Z}$ onto $\Delta_{Z} \in\left\{\mathbb{Z}, \mathbb{Z} \rtimes_{- \text {id }} \mathbb{Z} / 2\right\}$ since $\Gamma / Z$ is virtually cyclic; the kernel of $\hat{\mu}_{Z}$ is finite, and the restriction $\mu_{Z}$ of $\hat{\mu}_{Z}$ to $A / Z$ is injective. Set

$$
\begin{aligned}
\hat{v}_{Z} & :=\widehat{\mu}_{Z} \circ \hat{\xi}_{Z}: \Gamma \rightarrow \Delta_{Z}, \\
v_{Z}: & =\mu_{Z} \circ \xi_{Z}: A \rightarrow A_{Z} .
\end{aligned}
$$

Subject to some choice of finite generating set of $\Gamma$, there is a word metric $d_{\Gamma}$ on $\Gamma$. Let $\mathrm{ev}_{Z}: \Delta_{Z} \rightarrow \mathbb{R}$ be the map which is given by evaluating the natural $\Delta_{Z}$-action on $\mathbb{R}$ at 0 . Equip $\mathbb{R}$ with the simplicial structure whose set of vertices is $\{n / 2 \mid n \in \mathbb{Z}\}$.

Then there are positive constants $C_{1}$ and $C_{2}$ such that for every $F$-invariant, infinite cyclic subgroup $Z$ of $A$ and all $\gamma_{1}, \gamma_{2} \in \Gamma$ we have

$$
d^{\ell^{1}}\left(\operatorname{ev} Z \circ \hat{v}_{Z}\left(\gamma_{1}\right), \mathrm{ev}_{Z} \circ \hat{v}_{Z}\left(\gamma_{2}\right)\right) \leq C_{1} \cdot d_{\Gamma}\left(\gamma_{1}, \gamma_{2}\right)+C_{2}
$$

Fix $\varepsilon>0$ and choose two odd prime numbers $p_{1}$ and $p_{2}$ such that

$$
p_{i} \geq \frac{2 \cdot\left(C_{1}+C_{2}\right)}{\varepsilon}
$$

holds. Let $s:=p_{1} p_{2}$. Consider a subgroup $D \leq \Gamma_{s}$ which lies in $\mathcal{D} r$, and assume without loss of generality that $\operatorname{pr}_{s}(D)=F$. Pick a normal series $Q_{0} \unlhd D_{0} \unlhd D$ such that $Q_{0}$ is a $q_{0}$-group, $D / D_{0}$ is a $q_{1}$-group and $D_{0} / Q_{0}$ is a cyclic group of order prime to $q_{0}$ and $q_{1}$. 
If $q_{0} \in\left\{p_{1}, p_{2}\right\}$, say $q_{0}=p_{2}$, consider the projection $\pi: \Gamma_{s} \rightarrow \Gamma_{p_{1}}$. Then $\pi(D)$ is hyperelementary, and the argument on page 352 of [2] shows that $\pi(D) \cap A_{p_{1}}$ is cyclic.

If $q_{0} \notin\left\{p_{1}, p_{2}\right\}$, the group $D \cap A_{s}$ is $q_{1}$-hyperelementary. In case $q_{1}$ is one of $p_{1}$ and $p_{2}$, let us say that $q_{1}=p_{1}$. Otherwise, $D \cap A_{s}$ is even cyclic. In both cases, let $\pi: \Gamma_{s} \rightarrow \Gamma_{p_{2}}$ be the projection. Then $\pi(D) \cap A_{p_{2}}$ is cyclic.

In all cases we have considered, we have been able to find a prime $p \in\left\{p_{1}, p_{2}\right\}$ such that $\pi(D) \cap A_{p}$ is cyclic, where $\pi: \Gamma_{s} \rightarrow \Gamma_{p}$ is the canonical projection. Note that $\pi \circ \pi_{s}=\pi_{p}$. Set $\bar{D}:=\pi_{s}^{-1}(D)$. It follows that there is $j \in\{1,2\}$ such that

$$
\bar{D} \cap A \subset \pi_{s}^{-1}\left(\pi^{-1}(\pi(D))\right) \cap A=\pi_{p}^{-1}\left(\pi(D) \cap A_{p}\right) \subset \pi_{p}^{-1}\left(\pi_{p}\left(Z_{j}\right)\right) .
$$

Hence, $\xi_{Z_{j}}(\bar{D} \cap A) \subset p\left(A / Z_{j}\right)$. The remainder of the proof is as in [2, Lemma 3.15].

5.4 Proposition Let $\Gamma$ be a crystallographic group of rank at least 2 which does not contain a normal infinite cyclic subgroup. Let $\mathcal{F}$ be the family of all subgroups of $\Gamma$ whose virtual cohomological dimension is smaller than that of $\Gamma$ or which do not surject onto $F$ under pr.

Then $\Gamma$ is a Dress-Farrell-Hsiang group of bounded depth with respect to $\mathcal{F}$ (relative to an arbitrary finite generating set).

An important ingredient in the proof of Proposition 5.4 is the following mild generalization of the second part of Quinn [23, Proposition 2.4.2].

5.5 Lemma Let $\Gamma$ be an arbitrary crystallographic group. Let $p_{1}$ and $p_{2}$ be prime numbers which do not divide $|F|$, and let $r>0$ be a natural number. Set $s:=p_{1}^{r} p_{2}^{r}$. Suppose that $D \leq \Gamma_{s}$ is in $\mathcal{D r}$ such that $\operatorname{pr}_{s}(D)=F$ and $D \cap A_{s}$ is nontrivial.

Then there is $i \in\{1,2\}$ such that $\pi(D) \cap A / p_{i}^{r} A$ contains a nontrivial $F$-invariant cyclic subgroup, where $\pi$ is the canonical projection $\Gamma_{s} \rightarrow \Gamma_{p_{i}^{r}}$.

Proof Pick a normal series $Q_{0} \unlhd D_{0} \unlhd D$ such that $Q_{0}$ is a $q_{0}$-group, $D / D_{0}$ is a $q_{1}$-group, and $D_{0} / Q_{0}$ is a cyclic group whose order is coprime to $q_{0}$ and $q_{1}$.

Suppose $q_{0} \in\left\{p_{1}, p_{2}\right\}$, say $q_{0}=p_{1}$. Consider the projection $\pi: \Gamma_{s} \rightarrow \Gamma_{p_{1}^{r}}$. Then $\pi\left(Q_{0}\right)$ is trivial, so $\pi(D)$ is a hyperelementary subgroup of $\Gamma_{p_{1}^{r}}$ which surjects with a nontrivial kernel onto $F$. Apply [23, Proposition 2.4.2] to obtain the claimed result.

If $q_{0} \notin\left\{p_{1}, p_{2}\right\}$, then $Q_{0}$ injects into $F$ via $\operatorname{pr}_{s}$ and $D \cap A_{s}$ is $q_{1}$-hyperelementary. If also $q_{1} \notin\left\{p_{1}, p_{2}\right\}$, then $D \cap A_{s}=D_{0} \cap A_{s}$ is a nontrivial cyclic group. Let 
$p \in\left\{p_{1}, p_{2}\right\}$ be a divisor of the order of $D \cap A_{S}$, and let $\pi: \Gamma_{S} \rightarrow \Gamma_{p^{r}}$ be the projection. Then $\pi(D) \cap A_{p^{r}}$ is a nontrivial cyclic group. Since it equals the kernel of the surjection $\pi(D) \rightarrow F$, it is also $F$-invariant.

Now suppose $q_{1} \in\left\{p_{1}, p_{2}\right\}$, say $q_{1}=p_{1}$. Then $\operatorname{pr}_{s}\left(D_{0}\right)=F$.

In case $D_{0} \cap A_{s}$ is nontrivial, the projection $\pi: \Gamma_{s} \rightarrow \Gamma_{p_{2}^{r}}$ maps $D \cap A_{s}$ to the nontrivial cyclic group $\pi\left(D_{0} \cap A_{s}\right)$. This group is normal in $\pi(D)=\pi\left(D_{0}\right)$, and since $\pi\left(D_{0}\right)$ also surjects onto $F$, it is also $F$-invariant.

In case $D_{0} \cap A_{s}$ is trivial, $\mathrm{pr}_{s}$ restricts to an isomorphism $D_{0} \cong F$, and $D \cap A_{s}$ is a $q_{1}$-group isomorphic to $D / D_{0}$. It follows that $D \cong D_{0} \times D / D_{0}$. The projection $\pi: \Gamma_{s} \rightarrow \Gamma_{p_{1}^{r}}$ maps $D$ isomorphically to a subgroup of $\Gamma_{p_{1}^{r}}$; the kernel of the surjection $\pi(D) \rightarrow F$ is a nontrivial $q_{1}$-group (isomorphic to $D / D_{0}$ ). In particular, there is a nontrivial, normal and cyclic subgroup of the kernel. This subgroup is then also normal in $\pi(D)$ because $\pi(D)$ is the direct product of the kernel and $F$; in particular, it is $F$-invariant.

Proof of Proposition 5.4 (cf [2, Section 3.3]) Let $d_{\Gamma}$ be the word metric on $\Gamma$ with respect to some finite generating set $S$. Let ev: $\Gamma \rightarrow \mathbb{R}^{n}$ be the map that is given by evaluating the natural $\Gamma$-action on $\mathbb{R}^{n}$ at 0 . There are positive constants $C_{1}$ and $C_{2}$ such that

$$
d_{\text {euc }}\left(\operatorname{ev}\left(\gamma_{1}\right), \operatorname{ev}\left(\gamma_{2}\right)\right) \leq C_{1} \cdot d_{\Gamma}\left(\gamma_{1}, \gamma_{2}\right)+C_{2}
$$

for all $\gamma_{1}, \gamma_{2} \in \Gamma$.

Fix $\varepsilon>0$. Pick a simplicial structure on $\mathbb{R}^{n}$ such that $\mathbb{R}^{n}$ is a $\Gamma$-simplicial complex. Then there is $\delta>0$ such that

$$
d_{\text {euc }}\left(x_{1}, x_{2}\right) \leq \delta \Rightarrow d^{\ell^{1}}\left(x_{1}, x_{2}\right) \leq \varepsilon
$$

for all $x_{1}, x_{2} \in \mathbb{R}^{n}$.

Write $|F|=2^{k} \cdot l$ with $l$ a nonnegative odd natural number and $k$ a nonnegative natural number. Using Dirichlet's theorem [26, IV.4.1], pick two distinct prime numbers $p_{1}$ and $p_{2}$ such that

$$
p_{i} \equiv-1 \bmod 4 l, \quad p_{i} \geq \frac{C_{1}+C_{2}}{\delta}, \quad p_{i} \geq|F| .
$$

In particular, $|F|$ is coprime to both $p_{1}$ and $p_{2}$. Set $r:=\varphi(|F|)$, where $\varphi$ is Euler's $\varphi$-function. Then

$$
p_{i}^{r} \equiv 1 \bmod |F| \text {. }
$$

Put $s:=p_{1}^{r} p_{2}^{r}$. Note that the depth of $\Gamma_{s}$ can be uniformly bounded, independent of the choice of $p_{1}$ and $p_{2}$. 
Consider a subgroup $D \leq \Gamma_{s}$ which lies in $\mathcal{D} r$. We may assume that $\operatorname{pr}_{s}(D)=F$. Since $\Gamma$ contains no normal infinite cyclic subgroup, it follows from Lemma 5.5 in combination with [23, Proposition 2.4.2] that $D \cap A_{S}$ is trivial.

The proof continues as in [2, Section 3.3] from this point on to show that $\Gamma$ is a Dress-Farrell-Hsiang group of bounded depth with respect to $\mathcal{F}$.

Proposition 5.3 and Proposition 5.4 in conjunction with Lemma 5.2 prove Claim 4.4 by virtue of the transitivity principle. As was explained in Section 4, Claim 4.1 and Claim 4.3 follow from this.

\section{Transfer reducibility of irreducible special affine groups}

We shall now deal with Claim 4.5.

6.1 Theorem Every irreducible special affine group $\Gamma$ is a Dress-Farrell-Hsiang group of bounded depth with respect to the family of virtually finitely generated abelian groups.

The main technical ingredient for the proof of Theorem 6.1 is the following generalization of Bartels, Farrell and Lück [2, Section 4.4]:

6.2 Lemma There is a natural number $B$ such that for all natural numbers $o, v$ there are $r, s \in \mathbb{N}$ such that $s \equiv 1 \bmod o$ and for all $M \in \mathrm{GL}_{n}(\mathbb{Z})$ the following holds:

(1) The order of $\mathrm{GL}_{n}(\mathbb{Z} / s)$ divides $r$; so the semidirect product $(\mathbb{Z} / s)^{n} \rtimes_{M_{s}} \mathbb{Z} / r$ is defined, where $M_{s}$ denotes the reduction of $M$ modulo $s$. Let $\pi$ be the projection $(\mathbb{Z} / s)^{n} \rtimes_{M_{s}} \mathbb{Z} / r \rightarrow \mathbb{Z} / r$.

(2) The order of $(\mathbb{Z} / s)^{n} \rtimes_{M_{s}} \mathbb{Z} / r$ contains at most $B$ prime factors, counted with their multiplicities.

(3) All subgroups $G \leq(\mathbb{Z} / s)^{n} \rtimes_{M_{s}} \mathbb{Z} / r$ which lie in $\mathcal{D} r$ have one of the following properties:

(a) There is some $v^{\prime} \geq v$ which divides $s$ such that $v^{\prime} \equiv 1 \bmod o$ and $G \cap(\mathbb{Z} / s)^{n} \subset$ $v^{\prime}(\mathbb{Z} / s)^{n}$.

(b) $[\mathbb{Z} / r: \pi(G)] \geq v$. 
Proof Let us first explain how to obtain the bound on the number of prime factors in the order of $(\mathbb{Z} / s)^{n} \rtimes_{M_{s}} \mathbb{Z} / r$. Suppose $s$ is a product of pairwise distinct prime numbers $s=p_{1} \cdots p_{k}$. Then $\mathbb{Z} / s \cong \mathbb{Z} / p_{1} \times \cdots \mathbb{Z} / p_{k}$ as rings. Since the diagram

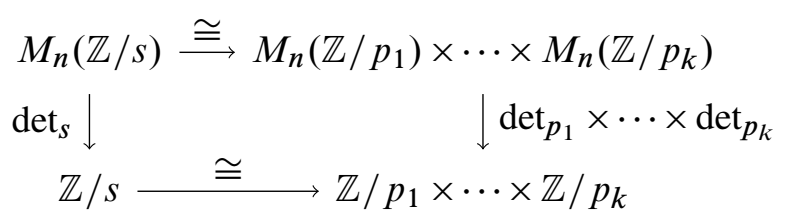

commutes and $\left(\mathbb{Z} / p_{1} \times \cdots \mathbb{Z} / p_{k}\right)^{*}=\left(\mathbb{Z} / p_{1}\right)^{*} \times \cdots \times\left(\mathbb{Z} / p_{k}\right)^{*}$, the top isomorphism induces an isomorphism

$$
\mathrm{GL}_{n}(\mathbb{Z} / s) \stackrel{\cong}{\rightarrow} \mathrm{GL}_{n}\left(\mathbb{Z} / p_{1}\right) \times \cdots \times \mathrm{GL}_{n}\left(\mathbb{Z} / p_{k}\right) .
$$

Define a polynomial $O_{n}(X) \in \mathbb{Z}[X]$ by

$$
O_{n}(X):=\left(X^{n}-1\right)\left(X^{n}-X\right) \cdots\left(X^{n}-X^{n-1}\right) .
$$

Then $O_{n}(p)$ is the order of $\mathrm{GL}_{n}(\mathbb{Z} / p)$ for any prime $p$, and consequently we have

$$
\left|\mathrm{GL}_{n}(\mathbb{Z} / s)\right|=O_{n}\left(p_{1}\right) \cdots O_{n}\left(p_{k}\right) .
$$

In order to bound the number of prime factors in this expression, we rely on the following generalization of Dirichlet's theorem:

6.3 Theorem (Miech [17]) Let $f(X) \in \mathbb{Z}[X]$ be a polynomial. Let $\rho$ and $\mu$ be natural numbers with $(\rho, \mu)=1$. Then there is a constant $K>0$ such that there are infinitely many primes $p$ with the property that $p \equiv \rho \bmod \mu$ and the number of prime factors of $f(p)$, counted with their multiplicities, is bounded by $K$.

Applying this theorem to $O_{n}(X)$ with $\rho=1$ and $\mu=o$, we obtain a positive number $K$ and an infinite set of primes $\mathcal{P}$ such that for all $p \in \mathcal{P}$, we have that $p \equiv 1 \bmod o$ and $O_{n}(p)$ has at most $K$ prime factors, counted with their multiplicities.

Let us now pick three distinct prime numbers $p_{1}, p_{2}$ and $p_{3}$ from $\mathcal{P}$ such that each of them is greater than $v$. Set $s:=p_{1} p_{2} p_{3}$ and $r:=\left|\mathrm{GL}_{n}(\mathbb{Z} / s)\right| \cdot s$. Then $s \equiv 1 \bmod o$ and the order of $M_{S}$ clearly divides $r$. Since

$$
\left|(\mathbb{Z} / s)^{n} \rtimes_{M_{s}} \mathbb{Z} / r\right|=s^{n} \cdot s \cdot\left|\mathrm{GL}_{n}(\mathbb{Z} / s)\right|=\left(p_{1} p_{2} p_{3}\right)^{n+1} \cdot\left|\mathrm{GL}_{n}(\mathbb{Z} / s)\right|,
$$

our preliminary considerations apply to show that the order of $(\mathbb{Z} / s)^{n} \rtimes_{M_{s}} \mathbb{Z} / r$ contains at most $B:=3(n+1)+3 K$ prime factors (counted with their multiplicities). 
What we have to check is that every subgroup $G \leq(\mathbb{Z} / s)^{n} \rtimes_{M_{s}} \mathbb{Z} / r$ which lies in $\mathcal{D} r$ has the desired properties. The proof is a direct adaptation of the arguments in [2, Lemma 4.18-4.21].

Fix a generator $t$ of $\mathbb{Z} / r$. Then every element of $(\mathbb{Z} / s)^{n} \rtimes_{M_{s}} \mathbb{Z} / r$ can be written in the form $v t^{j}$ for some $v \in(\mathbb{Z} / s)^{n}$ and some $j \in \mathbb{N}$.

Let us first consider the case of a subgroup $H \leq(\mathbb{Z} / s)^{n} \rtimes_{M_{s}} \mathbb{Z} / r$ which is cyclic $\bmod p$ for some prime $p$. Choose an extension $1 \rightarrow P \rightarrow H \rightarrow C \rightarrow 1$ such that $C$ is cyclic, $P$ is a $p$-group, and $p$ does not divide the order of $C$. Let $c \in C$ be a generator, and pick a preimage $v t^{j}$ under the epimorphism $H \rightarrow C$. Since $p$ does not divide $|C|$, the element $d:=c^{|P|}$ is another generator of $C$, and $\pi\left(\left(v t^{j}\right)^{|P|}\right)=d$. Write $\left(v t^{j}\right)^{|P|}=w t^{l}$. Set $x:=\left(w t^{l}\right)^{[\pi(H): \pi(P)]}$.

Suppose that $H \cap(\mathbb{Z} / s)^{n} \neq P \cap(\mathbb{Z} / s)^{n}$. By definition, $x$ lies in the kernel of $\left.\pi\right|_{H}$, which is $H \cap(\mathbb{Z} / s)^{n}$. Its image in $C$ is $d^{[\pi(H): \pi(P)]} \neq 0$, so $x$ does not lie in $P \cap(\mathbb{Z} / s)^{n}$. Let $s^{\prime}$ be the order of $x$. Then $s^{\prime}$ divides $|C|$; in particular, $p$ does not divide $s^{\prime}$. Note that $s^{\prime}$ divides $s$; write $s=\sigma \cdot s^{\prime}$. By the definition of $s$, the numbers $\sigma$ and $s^{\prime}$ are coprime. Let

$$
k:=\frac{\left|\mathrm{GL}_{n}(\mathbb{Z} / s)\right|}{\operatorname{gcd}\left(\left|\mathrm{GL}_{n}(\mathbb{Z} / s)\right|, s^{\prime}\right)} .
$$

Then $k \sigma$ and $s^{\prime}$ are still coprime. Consequently, $\left(w t^{l}\right)^{k \sigma[\pi(H): \pi(P)]}=x^{k \sigma} \neq 0$. On the other hand, one can compute as in [2, Lemma 4.19] that $\left(w t^{l}\right)^{s r^{\prime}}$ is the trivial element, so $s r^{\prime}$ does not divide $k \sigma[\pi(H): \pi(P)]$. Dividing by $k \sigma$ on both sides, we get $s^{\prime} \bar{r} \nmid[\pi(H): \pi(P)]$, where $\bar{r}$ is some natural number containing only prime factors which are also prime factors of $s^{\prime}$. Therefore, there is some $i \in\{1,2,3\}$ and a natural number $N \geq 1$ such that $p_{i}\left|s^{\prime}, p_{i}^{N}\right| s^{\prime} \bar{r}$, and $p_{i}^{N} \nmid[\pi(H): \pi(P)]$. Since $s^{\prime}$ is a divisor of $|H|$, the prime $p_{i}$ divides $|H|$.

It follows that $r=s r^{\prime}$ is divisible by $p_{i}^{N}$. As $p$ does not divide $s^{\prime}$, we must have $p \neq p_{i}$. In particular, $[\mathbb{Z} / r: \pi(P)]$ is also divisible by $p_{i}^{N}$. Since $[\pi(H): \pi(P)]$ is only divisible by primes which are also prime factors of $|C|$, the equality

$$
[\mathbb{Z} / r: \pi(H)] \cdot[\pi(H): \pi(P)]=[\mathbb{Z} / r: \pi(P)]
$$

implies that $p_{i}$ divides $[\mathbb{Z} / r: \pi(H)]$; in particular, $[\mathbb{Z} / r: \pi(H)] \geq p_{i} \geq v$.

Thus, we have shown that for every subgroup $H$ which is cyclic mod $p$ for some prime $p$, there is an extension $1 \rightarrow P \rightarrow H \rightarrow C \rightarrow 1$ with $C$ a cyclic group, $P$ a $p$-group such that $p \nmid|C|$, and one of the following statements is true:

- $H \cap(\mathbb{Z} / s)^{n}=P \cap(\mathbb{Z} / s)^{n}$.

- There is $i \in\{1,2,3\}$ such that $p \neq p_{i}, p_{i}|| H \mid$ and $p_{i} \mid[\mathbb{Z} / r: \pi(H)]$. 
We are now going to use this to show the actual claim. So let $G \in \mathcal{D r}$ be a subgroup of $(\mathbb{Z} / s)^{n} \rtimes_{M_{s}} \mathbb{Z} / r$. Pick an extension $1 \rightarrow H \rightarrow G \rightarrow Q \rightarrow 1$ such that $H$ is cyclic $\bmod p$ for some prime $p$ and $Q$ is a $q$-group. If $p \neq q$, we may assume that $q$ does not divide $|H|$. Note that both $\left[G \cap(\mathbb{Z} / s)^{n}: H \cap(\mathbb{Z} / s)^{n}\right]$ and $[\pi(G): \pi(H)]$ are $q$-powers. Choose an extension $1 \rightarrow P \rightarrow H \rightarrow C \rightarrow 1$ with the properties we had just discussed.

Assume first that $H \cap(\mathbb{Z} / s)^{n}=P \cap(\mathbb{Z} / s)^{n}$. Then $\left|G \cap(\mathbb{Z} / s)^{n}\right|=p^{k} q^{l}$ for some natural numbers $k$ and $l$. Choose $i$ such that $p \neq p_{i} \neq q$. Let $\gamma$ be a generator of $\mathbb{Z} / s$, and let $\left(g_{1}, \ldots, g_{n}\right) \in G \cap(\mathbb{Z} / s)^{n}$ be an arbitrary element. There are natural numbers $a_{j}$ such that $g_{j}=\gamma^{a_{j}}$. It follows that $\gamma^{a_{j} p^{k} q^{l}}=0$, so $a_{j}$ is divisible by $p_{i}$. This shows that $G \cap(\mathbb{Z} / s)^{n} \subset p_{i}(\mathbb{Z} / s)^{n}$. Note that by our initial choice of $p_{i} \in \mathcal{P}$, it is automatically true that $p_{i} \geq v, p_{i}$ divides $s$, and $p_{i} \equiv 1 \bmod o$.

Consider now the case that $H \cap(\mathbb{Z} / s)^{n} \neq P \cap(\mathbb{Z} / s)^{n}$, so there is some $i$ such that $p \neq p_{i}, p_{i}|| H \mid$ and $p_{i} \mid[\mathbb{Z} / r: \pi(H)]$. We must also have $q \neq p_{i}$. Since

$$
[\mathbb{Z} / r: \pi(G)] \cdot[\pi(G): \pi(H)]=[\mathbb{Z} / r: \pi(H)],
$$

the prime $p_{i}$ must divide $[\mathbb{Z} / r: \pi(G)]$, so $[\mathbb{Z} / r: \pi(G)] \geq p_{i} \geq v$. This finishes the proof.

Proof of Theorem 6.1 We have to introduce some additional notation. Recall that we can write $\Gamma$ as an extension $1 \rightarrow \Theta \rightarrow \Gamma \rightarrow \Delta \rightarrow 1$ of a crystallographic group $\Theta$ by $\Delta \in\left\{\mathbb{Z}, D_{\infty}\right\}$. Let $A$ be the unique normal, free abelian subgroup of $\Theta$ which equals its own centralizer. Set $Q:=\Gamma / A$. As before, set $A_{s}:=A / s A$ for any positive integer $s$. Note that $A_{s}$ is isomorphic to $(\mathbb{Z} / s)^{n}$. Moreover, the virtually cyclic group $Q$ has a normal, infinite cyclic subgroup $C \leq Q$. Let $F$ be the finite quotient $Q / C$, and denote for any positive integer $r$ the quotient $Q / r C$ by $Q_{r}$. As described in [2, page 359], there is a certain semidirect product $A_{s} \rtimes_{\rho_{r, s}} Q_{r}$ whenever $r$ divides the order of $\operatorname{aut}\left(A_{s}\right)$, and one can construct a projection map $\pi_{r, s}: \Gamma \rightarrow A_{s} \rtimes_{\rho_{r, s}} Q_{r}$. If $G \leq A_{s} \rtimes_{\rho_{r, s}} Q_{r}$ is any subgroup, write $\bar{G}$ for the preimage $\alpha_{r, s}^{-1}(\bar{G})$.

First, we observe that given $\tau \in \mathbb{N}$, there are natural numbers $r, s \in \mathbb{N}$ such that

(1) $s \equiv 1 \bmod \left|H^{2}(Q ; A)\right|$.

(2) $\left|\operatorname{aut}\left(A_{S}\right)\right| \mid r$.

(3) For every subgroup $G \leq A_{s} \rtimes_{\rho_{r, s}} Q_{r}$ which lies in $\mathcal{D r}$, one of the following holds:

- The order of $H^{1}(\operatorname{pr}(\bar{G}) ; A)$ and $H^{2}(\operatorname{pr}(\bar{G}) ; A)$ is finite and there is $k \in \mathbb{N}$ such that $k$ divides $s, k$ is at least $\tau, k \equiv 1 \bmod \left|H^{1}(\operatorname{pr}(\bar{G}) ; A)\right|, k \equiv$ $1 \bmod \left|H^{2}(Q ; A)\right|, k \equiv 1 \bmod \left|H^{2}(\operatorname{pr}(\bar{G}) ; A)\right|$, and $\bar{G} \cap A \subset k A$.

- $[D: \pi(\operatorname{pr}(\bar{G}))] \geq \tau$. 
This is nontrivial, but one can copy the proof of [2, Lemma 4.22] verbatim, noticing that the only part of the proof which is specific to hyperelementary subgroups is the invocation of [2, Proposition 4.10], which we can replace by Lemma 6.2. Observe that

$$
\left|A_{s} \rtimes_{\rho_{r, s}} Q_{r}\right|=\left|A_{s}\right| \cdot\left|Q_{r}\right|=\left|(\mathbb{Z} / s)^{n}\right| \cdot|\mathbb{Z} / r| \cdot|F| .
$$

Since we used Lemma 6.2 to choose $r$ and $s$, we know that $\left|(\mathbb{Z} / s)^{n}\right| \cdot|\mathbb{Z} / r|$ contains at most $B$ prime factors. Moreover, the order of $F=Q / C$ does not depend on any of the choices we made, and thus always contains the same number of prime factors. In total, this gives us a uniform bound on the number of prime factors occurring in the order of $\left|A_{s} \rtimes_{\rho_{r, s}} Q_{r}\right|$, counted with their multiplicities, and thus on the depth of $A_{s} \rtimes_{\rho_{r, s}} Q_{r}$.

Now the proof can be finished by arguing precisely as in the proof of [2, Proposition 4.41].

\section{Appendix: On the proof of Oliver's theorem}

As promised, we are now going to review the proof of Oliver's Theorem 2.9 to show the existence of the function bd. The outline of the proof is basically that of Oliver [19], with some additional input from Oliver [21]. However, we will deviate from the treatment in [19] at the end to get a better grip on the dimension bound.

Let $G$ be a finite group throughout. Let $\Omega(G)$ be the Burnside ring of $G$. We think about elements in $\Omega(G)$ as equivalence classes of finite $G-\mathrm{CW}$-complexes, where the relevant equivalence relation $\sim_{\chi}$ is the following: Two finite $G-\mathrm{CW}$-complexes $X$ and $Y$ are $\chi$-equivalent, $X \sim_{\chi} Y$, if and only if $\chi\left(X^{H}\right)=\chi\left(Y^{H}\right)$ for all subgroups $H \leq G$, where $\chi$ denotes the Euler characteristic of a finite $\mathrm{CW}$-complex. The disjoint union and product operations induce the ring structure on $\Omega(G)$. See Oliver [20, page 90] and tom Dieck [10] for more information on this description of $\Omega(G)$.

Let $\Delta(G) \subset \Omega(G)$ be the subset given by

$$
\Delta(G):=\left\{\begin{array}{l|l}
x \in \Omega(G) & \begin{array}{l}
\text { There is a finite contractible } G-\mathrm{CW} \text {-complex } \\
X \text { with } x=[X]-1 .
\end{array}
\end{array}\right\} .
$$

As Oliver observed in [20, page 90], this is an ideal in $\Omega(G)$. Let $\operatorname{gh}_{G}: \Omega(G) \rightarrow \mathbb{Z}$ be the "ghost map" which sends $[X]$ to $\chi\left(X^{G}\right)$. Then the image of $\Delta(G)$ under $\operatorname{gh}_{G}$ is an ideal in $\mathbb{Z}$; we let $n_{G}$ denote the unique nonnegative generator. One easily observes that $n_{G}=1$ if there is a finite contractible $G-\mathrm{CW}$-complex without a global fixed point.

Another important concept is that of a resolving function: 
A.4 Definition [19, bottom of page 159] Let $\mathcal{S}(G)$ denote the poset of subgroups of $G$. A function $\varphi: \mathcal{S}(G) \rightarrow \mathbb{Z}$ is a resolving function if the following holds:

- $\varphi$ is constant on conjugacy classes of subgroups.

- For all $H \leq G$, the order of the Weyl group $\left[N_{G}(H): H\right]$ divides $\varphi(H)$.

- If $H \in \mathcal{C} y c_{p}$ for some prime $p$, then $\sum_{K \supset H} \varphi(K)=0$.

Every finite, contractible $G-\mathrm{CW}$-complex $X$ gives rise to a resolving function $\varphi_{X}$ [19, Proposition 2 and Lemma 2]. The set

$$
\{\varphi(G) \mid \varphi \text { is a resolving function for } G\} \subset \mathbb{Z}
$$

forms a subgroup, and we let $r_{G}$ denote the unique nonnegative generator of this group. If $[X]-1$ is a preimage of $n_{G}$ with respect to $\mathrm{gh}_{G}$, then $n_{G}=\chi\left(X^{G}\right)-1=\varphi_{X}(G)$. It follows that $r_{G}$ is always a divisor of $n_{G}$.

A.5 Theorem (cf [19, Theorem 2]) If $r_{G}=1$ and $G$ is not a $p$-group for any prime $p$, then there is a finite, contractible $G-C W$-complex $X$ without global fixed point whose dimension is bounded by $4 \cdot d(G)+2$.

As a first step towards Theorem A.5, one constructs a $G$-resolution $Y$, ie a finite, $n$-dimensional and $(n-1)$-connected $G-\mathrm{CW}$-complex $Y$ with $Y^{G}=\varnothing$ such that $H_{n}(Y ; \mathbb{Z})$ is a finitely generated projective $\mathbb{Z}[G]$-module. In addition, we will see that the dimension $n$ of $Y$ can be bounded by $2 \cdot d(G)$.

To keep track of how the construction proceeds, we try to make the induction as explicit as possible. Let $\mathcal{S}(G)$ be again the poset (with respect to $\supseteq$ ) of subgroups of $G$. Define the rank of a subgroup $H \leq G$ to be

$$
\operatorname{rk}(H):=\max \{\operatorname{rk}(K) \mid K \in \mathcal{S}(G), K \supsetneq H\}+1,
$$

where we let $\max \varnothing=0$. Note that the depth of $G$ is precisely $\operatorname{rk}\left(\left\{1_{G}\right\}\right)$, and that conjugate subgroups have equal rank. For each $r \leq d(G)$, choose a linear order $\preccurlyeq r$ on the set of subgroups of rank $r$. Then

$$
H \preccurlyeq H^{\prime} \Longleftrightarrow \operatorname{rk}(H)<\operatorname{rk}\left(H^{\prime}\right) \quad \text { or } \quad\left(\operatorname{rk}(H)=\operatorname{rk}\left(H^{\prime}\right) \text { and } H \preccurlyeq_{\operatorname{rk}(H)} H^{\prime}\right)
$$

defines a linear order on $\mathcal{S}(G)$. We write $H \prec H^{\prime}$ if $H \preccurlyeq H^{\prime}$ and $H \neq H^{\prime}$.

The construction of $Y$ proceeds inductively along the finite linear order $(\mathcal{S}(G) \backslash\{1\}, \prec)$. By assumption, we may choose a resolving function $\varphi$ on $G$ with $\varphi(G)=-1$. Then we claim that for every $H \in \mathcal{S}(G) \backslash\{1\}$, there is a finite $G-\mathrm{CW}$-complex $Y(H)$ such that the following holds: 
(1) The complex $Y(H)$ contains only cells of type $G / K$ for $K \neq G$ and $K \preccurlyeq H$; in particular, it has no global fixed point.

(2) The dimension of $Y(H)$ is bounded by $2 \cdot \operatorname{rk}(H)$.

(3) For every $K \preccurlyeq H$, the dimension of the $K$-fixed point set $Y(H)^{K}$ is bounded by $2 \cdot \operatorname{rk}(K)$.

(4) $\chi\left(Y(H)^{K}\right)=1+\sum_{K^{\prime} \supseteq K} \varphi\left(K^{\prime}\right)$ for all $K \preccurlyeq H$.

(5) If $K \preccurlyeq H$ is a $p$-group for some prime $p$, the $K$-fixed points $Y(H)^{K}$ are $\mathbb{Z} / p$-acyclic.

For the start of the induction, we can set $Y(G):=\varnothing$. Suppose that $Y\left(H^{-}\right)$has been constructed for some subgroup $H^{-} \leq G$. Let $H$ be the immediate successor of $H^{-}$. If there is some conjugate $\mathrm{gHg}^{-1}$ of $H$ such that $\mathrm{gHg}^{-1} \prec H$, we may set $Y(H):=Y\left(H^{-}\right)$. Suppose that no conjugate of $H$ is $\prec-$ smaller than $H$.

Assume first that $H$ is no $p$-group. If $n=0$, set $Y(H):=Y\left(H^{-}\right)$. Otherwise, we can add cells of type $G / H$ in sufficiently low dimensions to enforce condition (4) without affecting the other properties.

So suppose now that $H$ is a $p$-group for some prime $p$. Attach successively cells of type $G / H$ to $Y\left(H^{-}\right)$to construct a $G-\mathrm{CW}$-complex $Y^{\prime}$ whose $H$-fixed point set $\left(Y^{\prime}\right)^{H}$ has dimension bigger than the $K$-fixed point sets $\left(Y^{\prime}\right)^{K}$ for all $K \supsetneq H$ and is $\left(\operatorname{dim}\left(Y^{\prime}\right)-1\right)$-connected. We can arrange that $\operatorname{dim}\left(Y^{\prime}\right) \leq 2 \cdot \operatorname{rk}(H)-1$. By the argument on page 162 of [19], the top-dimensional homology $H_{\operatorname{dim}\left(Y^{\prime}\right)}(Y ; \mathbb{Z} / p)$ is free. This allows us to glue on a set of $\left(\operatorname{dim}\left(Y^{\prime}\right)+1\right)$-cells of type $G / H$ to obtain a finite $G-\mathrm{CW}$-complex $Y(H)$ whose dimension is bounded by $2 \cdot \operatorname{rk}(H)$ and which is $\mathbb{Z} / p$-acyclic. One checks that $Y(H)$ has all other desired properties.

At the end of the induction, we have a finite $G-\mathrm{CW}-$ complex $Y_{0}$ which has no global fixed point, whose dimension $n^{\prime}$ is bounded by $2 \cdot d(G)-2$, whose fixed-point sets under nontrivial $p$-groups are $\mathbb{Z} / p$-acyclic and which satisfies $\chi\left(Y_{0}^{H}\right)=1+\sum_{K \supseteq H} \varphi(K)$ for all $H \neq 1$.

By another induction along the skeleta, we can glue on free $G$-cells to produce an $\left(n^{\prime}+1\right)$-dimensional and $n^{\prime}$-connected $G-\mathrm{CW}$-complex $Y$ which has no global fixed point and whose top-dimensional homology $H_{n^{\prime}+1}(Y ; \mathbb{Z})$ is finitely generated and projective as a $\mathbb{Z}[G]$-module (see [19, Proof of Theorem 2] for the last claim). Setting $n:=n^{\prime}+1$, we have found a $G$-resolution.

Theorem A.5 can be derived from the existence of a $G$-resolution $Y$ as follows: Take the join $X^{\prime}:=Y * Y$. We will think about the join of two spaces $Z$ and $Z^{\prime}$ as 
$\left(C(Z) \times Z^{\prime}\right) \cup_{Z \times Z^{\prime}}\left(Z \times C\left(Z^{\prime}\right)\right)$ via the homeomorphism

$$
\begin{aligned}
Z * Z^{\prime} & \rightarrow\left(C(Z) \times Z^{\prime}\right) \cup_{Z \times Z^{\prime}}\left(Z \times C\left(Z^{\prime}\right)\right), \\
\left(z, t, z^{\prime}\right) & \mapsto \begin{cases}\left(z,\left(1-2 t, z^{\prime}\right)\right) \in Z \times C\left(Z^{\prime}\right) \quad t \leq \frac{1}{2}, \\
\left((z, 2 t-1), z^{\prime}\right) \in C\left(Z^{\prime}\right) \times Z & t \geq \frac{1}{2} .\end{cases}
\end{aligned}
$$

This description makes it obvious that $X^{\prime}$ is a $G-\mathrm{CW}-$ complex without a global fixed point whose dimension can be bounded by $2 n+1 \leq 4 \cdot d(G)+1$. Moreover, we can use the given decomposition to apply the Seifert-van Kampen theorem, and then proceed by induction with the Hurewicz theorem and Mayer-Vietoris sequence for homology to show that $X^{\prime}$ is $2 n$-connected. The isomorphisms

$$
H_{2 n+1}\left(X^{\prime} ; \mathbb{Z}\right) \stackrel{\cong}{\rightarrow} H_{2 n}(Y \times Y ; \mathbb{Z}) \stackrel{\cong}{\longleftarrow} H_{n}\left(X^{\prime} ; \mathbb{Z}\right) \otimes_{\mathbb{Z}} H_{n}\left(X^{\prime} ; \mathbb{Z}\right),
$$

which can be assembled from the Mayer-Vietoris sequence and the Künneth Theorem, are both $\mathbb{Z}[G]$-linear. We can now invoke Oliver and Segev [18, Proposition C.3] to deduce that $H_{2 n+1}\left(X^{\prime}, \mathbb{Z}\right)$ is a stably free $\mathbb{Z}[G]$-module. Hence, we can add free $(2 n+1)-$ and $(2 n+2)$-cells to produce a finite, contractible $G-\mathrm{CW}$-complex $X$ without global fixed point whose dimension is bounded by $4 \cdot d(G)+2$.

A.6 Corollary Suppose $G$ is not a $p$-group for any prime $p$. Then the following are equivalent:

(1) $r_{G}=1$.

(2) There is a finite, contractible $G-C W$-complex $X$ with $X^{G}=\varnothing$ whose dimension is bounded by $4 \cdot d(G)+2$.

(3) $n_{G}=1$.

Since Oliver has shown in [19, Theorem 5] that $r_{G}=1$ if and only if $G \notin \mathcal{D} r$, Theorem 2.9 follows from the well-known fact that every finite $G-\mathrm{CW}$-complex is $G$-homotopy equivalent to a finite $G$-simplicial complex of equal dimension, see eg Oliver and Segev [18, Proposition A.4].

\section{References}

[1] A Bartels, On proofs of the Farrell-Jones conjecture (2014) arXiv:1210.1044

[2] A Bartels, F T Farrell, W Lück, The Farrell-Jones conjecture for cocompact lattices in virtually connected Lie groups, J. Amer. Math. Soc. 27 (2014) 339-388 MR3164984

[3] A Bartels, W Lück, Induction theorems and isomorphism conjectures for $K$ - and L-theory, Forum Math. 19 (2007) 379-406 MR2328114 
[4] A Bartels, W Lück, The Borel conjecture for hyperbolic and CAT(0)-groups, Ann. of Math. 175 (2012) 631-689 MR2993750

[5] A Bartels, W Lück, The Farrell-Hsiang method revisited, Math. Ann. 354 (2012) 209-226 MR2957625

[6] A Bartels, W Lück, Geodesic flow for CAT(0)-groups, Geom. Topol. 16 (2012) 1345-1391 MR2967054

[7] A Bartels, W Lück, H Reich, The K-theoretic Farrell-Jones conjecture for hyperbolic groups, Invent. Math. 172 (2008) 29-70 MR2385666

[8] A Bartels, W Lück, H Reich, H Rüping, $K$ - and L-theory of group rings over $G L_{n}(\mathbf{Z})$, Publ. Math. Inst. Hautes Études Sci. 119 (2014) 97-125 MR3210177

[9] G Carlsson, E K Pedersen, Controlled algebra and the Novikov conjectures for Kand L-theory, Topology 34 (1995) 731-758 MR1341817

[10] T tom Dieck, The Burnside ring of a compact Lie group, I, Math. Ann. 215 (1975) 235-250 MR0394711

[11] F T Farrell, W C Hsiang, The topological-Euclidean space form problem, Invent. Math. 45 (1978) 181-192 MR0482771

[12] F T Farrell, W C Hsiang, The Whitehead group of poly-(finite or cyclic) groups, J. London Math. Soc. 24 (1981) 308-324 MR631942

[13] F T Farrell, W C Hsiang, Topological characterization of flat and almost flat Riemannian manifolds $M^{n}(n \neq 3,4)$, Amer. J. Math. 105 (1983) 641-672 MR704219

[14] F T Farrell, L E Jones, Isomorphism conjectures in algebraic K-theory, J. Amer. Math. Soc. 6 (1993) 249-297 MR1179537

[15] H Kammeyer, W Lück, H Rüping, The Farrell-Jones conjecture for arbitrary lattices in virtually connected Lie groups, preprint (2015) arXiv:1401.0876 to appear in Geom. Topol.

[16] W Lück, H Reich, The Baum-Connes and the Farrell-Jones conjectures in K-and $L$-theory, from: "Handbook of $K$-theory, Vol. 1, 2", Springer, Berlin (2005) 703-842

[17] R J Miech, Primes, polynomials and almost primes, Acta Arith. 11 (1965) 35-56 MR0179139

[18] B Oliver, Y Segev, Fixed point free actions on $\mathbf{Z}$-acyclic 2-complexes, Acta Math. 189 (2002) 203-285 MR1961198

[19] R Oliver, Fixed-point sets of group actions on finite acyclic complexes, Comment. Math. Helv. 50 (1975) 155-177 MR0375361

[20] R Oliver, Smooth compact Lie group actions on disks, Math. Z. 149 (1976) 79-96 MR0423390 
[21] R Oliver, Group actions on disks, integral permutation representations, and the Burnside ring, from: "Algebraic and geometric topology, Part 1", (R J Milgram, editor), Proc. Sympos. Pure Math. 32, Amer. Math. Soc. (1978) 339-346 MR520510

[22] E K Pedersen, C A Weibel, A nonconnective delooping of algebraic $K$-theory, from: "Algebraic and geometric topology", (A Ranicki, N Levitt, F Quinn, editors), Lecture Notes in Math. 1126, Springer, Berlin (1985) 166-181 MR802790

[23] F Quinn, Algebraic K-theory over virtually abelian groups, J. Pure Appl. Algebra 216 (2012) 170-183 MR2826431

[24] A Ranicki, Lower K-and L-theory, London Math. Soc. Lecture Note Series 178, Cambridge Univ. Press (1992) MR1208729

[25] W R Scott, Group theory, 2nd edition, Dover, New York (1987) MR896269

[26] J-P Serre, A course in arithmetic, Graduate Texts in Mathematics 7, Springer, New York (1973) MR0344216

[27] $R$ G Swan, Induced representations and projective modules, Ann. of Math. 71 (1960) 552-578 MR0138688

[28] C Wegner, The K-theoretic Farrell-Jones conjecture for CAT(0)-groups, Proc. Amer. Math. Soc. 140 (2012) 779-793

[29] C Wegner, The Farrell-Jones conjecture for virtually solvable groups, preprint (2015) arXiv: 1308.2432

Mathematisches Institut, Westfälische Wilhelms-Universität Münster

Einsteinstr. 62, D-48149 Münster, Germany

christoph.winges@wwu.de

Received: 9 October $2014 \quad$ Revised: 5 March 2015 